Revista de Psicología Vol. 33 (1), 2015 (ISSN 0254-9247)

\title{
Programas de tutoría y mentoría en universidades brasileñas: un estudio bibliométrico ${ }^{1}$
}

\author{
Patrícia Albanaes ${ }^{2}$, Frederico Marques de Sousa Soares ${ }^{3}$ y \\ Marucia Patta Bardagi ${ }^{4}$ \\ Universidade Federal de Santa Catarina - Brasil
}

\begin{abstract}
Los procesos de tutoría y mentoría académicas se han descrito como herramientas relevantes para el desarrollo global y la integración académica del estudiante universitario. Este artículo realiza una revisión bibliométrica de los programas de tutoría y mentoría en universidades brasileñas, entre 1998 y 2013, con el fin de mapear las iniciativas existentes, su configuración, objetivos y principales resultados. Después de la revisión del Banco de Tesis de CAPES, SciELO, INDEXPSI y BVS-Psi, fueron encontradas 31 publicaciones sobre el tema. La mayoría de los trabajos se refieren a estudios cualitativos en el área de la salud desarrollados en el sureste y noreste del país en instituciones públicas. Los resultados indicaron una evaluación positiva de las experiencias de mentoría y tutoría, pero fueron señalados algunos aspectos a considerar en futuros proyectos con el fin de mejorar los resultados.

Palabras clave: tutoría, mentoría, estudiante universitario, integración académica
\end{abstract}

\section{Tutoring and mentoring programs in Brazilian universities: A bibliometric study}

The university's processes of tutoring and mentoring have been described as relevant tools for overall development and academic integration of the university student and overall development of the university student, and academic integration. This study conducted

1 Los autores agradecen a CNPq por el apoyo al proyecto a través de la concesión de una beca Pibic/UFSC y del financiamiento a través de la Convocatoria Ciencias Humanas Llamada MCTI /CNPq /MEC/CAPES No 07/2011.

2 Estudiante de piscología de la UFSC. Becaria Pibic/Cnpq/UFSC. Dirección postal: sala 14A, Departamento de Psicología CFH/UFSC, Campus João David Ferreira Lima, Bairro Trindade, Florianópolis, SC, Brasil. CEP 88040-900. Contacto: patricia.albanaes@gmail.com

3 Estudiante de psicología de la UFSC. Becario Pibic voluntario. Dirección postal: sala 14A, Departamento de Psicología CFH/UFSC, Campus João David Ferreira Lima, Bairro Trindade, Florianópolis, SC, Brasil. CEP 88040-900. Contacto: fred_marques_10@hotmail.com

4 Doctora por la UFRGS. Profesora del Departamento y del Programa de Posgraduación en Psicología de la UFSC. Dirección postal: sala 14A, Departamento de Psicología CFH/UFSC, Campus João David Ferreira Lima, Bairro Trindade, Florianópolis, SC, Brasil. CEP 88040900. Contacto: marucia.patta@ufsc.br 
a bibliometric review about tutoring and mentoring programs in Brazilian universities between 1998 and 2013 in order to map existing initiatives, configuration, objectives, key findings and relevance. After consulting CAPES Repositorium, SciELO, INDEXPsi and BVS-Psi, 31 papers were analyzed. Most studies in the area of health were conducted in public institutions with qualitative methods, in the southeast and northeast of Brazil. Results indicated positive evaluation of the experiences, and point to aspects to be considered in future projects.

Keywords: Mentoring, tutoring, university student, academic integration 
Los procesos de tutoría y mentoría son descritos en la literatura como importantes herramientas en el proceso formativo de la Enseńanza Superior. De manera general, esas estrategias se caracterizan por una diversidad de acciones que benefician al estudiante en los aspectos propios del proceso formativo, relacionados con diferentes esferas de la formación académica, vocacional, personal y social. La literatura internacional, tanto de origen anglosajona, como ibérica y latina, es vasta, y comprende estudios teóricos y empíricos sobre estos procesos. Particularmente en Brasil, la discusión sobre este tópico es incipiente. Con el propósito de llenar estos vacíos epistemológicos, el presente estudio parte de una descripción teórica sobre el origen y la caracterización de los programas de tutoría y mentoría universitarias; luego, ofrece una revisión bibliométrica sobre las experiencias acumuladas con relación a este tema, en las instituciones de Enseñanza Superior del país.

\section{Origen, definiciones y objetivos de los programas de tutoría y mentoría universitarias}

Geib, Krahl, Poletto y Silva (2007), en un estudio de revisión sobre la historia de la tutoría académica, afirman que a inicios del siglo XIII los programas de tutoría surgieron en las universidades, pero no es hasta la primera mitad del siglo XX en que se observa una significativa expansión de dichas prácticas, tanto en los países desarrollados como en aquellos en proceso de desarrollo. Rodríguez (2004) y Flores, Kury y Abreu (2011), aseguran que el origen de la tutoría universitaria se encuentra en la propia concepción de la universidad y, en este sentido, distinguen tres modelos de Enseñanza Superior: el modelo académico (vinculado a la tradición alemana), en el cual las actividades tutorales tienen como objetivo el dominio de los conocimientos académicos de 
sus estudiantes; y, por último, el modelo de desarrollo profesional, en el que las actividades tutorales incluyen el apoyo y la capacitación en correspondencia con la profesión y el mercado de trabajo.

A pesar de la existencia de múltiples definiciones en la literatura sobre el concepto de tutoría universitaria, se aprecia una convergencia entre los teóricos al referirse a esta como un conjunto de acciones desarrolladas por docentes, con el propósito de contribuir al aprendizaje y al proceso global de formación profesional del alumno (Aguilar, Rodríguez \& Bugdug, 2012; Budge, 2006; Crisp \& Cruz, 2009; Díaz, 2004; Eby, Allen, Evans, Ng \& DuBois, 2008; Flores et al., 2011; Gómez-Collado, 2012). Frente a las diferentes perspectivas y modelos de intervención tutorial en las instituciones de Enseñanza Superior (Crisp \& Cruz, 2009), el desafío del tutor se centra en las herramientas que pueda ofrecer para que el tutorado mejore su desempeño y perfeccione su aprendizaje de manera autorregulada (López, Salazar, García \& Medina, 2012), a través de acciones como orientar, guiar, aconsejar, informar, acompañar y escuchar a su tutorado.

El concepto y las delimitaciones de la actuación de programas de mentoría no aparecen consensuados en la literatura, como ocurre también con la tutoría. Por ejemplo, en un estudio de revisión, Crisp y Cruz (2009) encontraran más de 500 definiciones diversas referidas a la modalidad de mentoring. Además, en muchos casos la mentoría aparece como sinónimo de tutoría. En la mentoría, está presente también el aspecto relacional recíproco, en el que el objetivo principal no deja de ser el crescimiento del estudiante. El modelo propuesto por Kram (1983) defiende al mentor como un sujeto de vasto conocimiento y experiencia, que confiere un soporte de carrera y apoyo psicosocial al mentorado. Entre tanto, en relación con el contexto académico, si la tutoría es una acción esencialmente docente, la mentoría puede, por su parte, ser realizada por los propios estudiantes. Erlich (2010) afirma que los mentores no tienen que tener necesariamente un estatus, ni poseer un grado jerárquico o autoridad mayor. De acuerdo con el autor, lo que importa es que el mentor tenga el conocimiento y la experiencia suficiente para ofrecércelos a sus estudiantes. 
En este sentido, Mundina, Pombo y Ruiz (2007), indican que la mentoría se apoya en una relación personal de espontaneidad, cuando el mentor y el mentorado son estudiantes de la misma institución. Los mentores asumen un importante papel en el auxilio a los estudiantes, especialmente a los de nuevo ingreso en la universidad. Marinho-Araujo y Bisinoto (2011), por ejemplo, afirman que los mentores acogen y acompañan a los nuevos discípulos, ofreciéndoles atención especializada y sistemática, con el objetivo de facilitar su inserción en el medio académico.

Según destacan Simón, Flores, Fernandez y Figueira (2008), así como López y colaboradores (2012), la acción tutorial puede comprender el conjunto de objetivos siguientes: (a) orientar al alumno en el conocimiento de la universidad para una mayor integración en el nuevo contexto universitario; (b) desarrollar la capacidad de estudiar y aprender con mayor eficiencia; y (c) informar al alumno sobre cuestiones académicas y/o profesionales. El primer aspecto converge con lo señalado por Díaz (2004) como tutoría formativa, con los objetivos centrados especialmente en los aspectos emocionales y afectivos durante la adaptación del estudiante, en los primeros semestres de ingreso a la institución. El segundo aspecto hace referencia a la tutoría académica, cuyo objetivo específico consiste en auxiliar en el proceso de enseñanza y aprendizaje en la trayectoria formativa del alumno. López y colaboradores (2012) destacan otros objetivos posibles de intervención en los programas de tutoría, tales como: reducir la ansiedad en la preparación para los exámenes, opciones de trayectoria, feedback a la estabilidad emocional y actitudes referidas al campo profesional, así como el vínculo a las actividades extracurriculares que puedan propiciar al máximo la calidad del proceso formativo.

Por su parte, Rubio y Martínez (2012) subrayan la importancia de los aspectos de integración y aprendizaje de la tutoría, entre ellos: la tutoría personal, centrada en los aspectos emocionales y afectivos que pueden interferir en el proceso de enseñanza-aprendizaje; la tutoría social, destinada a cuestiones referentes a la beca de estudios, condiciones de permanencia en las universidades y movilidad estudiantil; y la 
tutoría administrativa, relativa a los requisitos administrativos, matrícula, uso de banco de datos, biblioteca, etc.

Uno de los autores más citados internacionalmente, Kram (1983), presenta una perspectiva en relación con el proceso de mentoría, vinculando dos categorías amplias de funciones diversas y complementarias, que son: a) funciones de carrera (patrocinio y padrinazgo, exposición y visibilidad, coaching/entrenamiento, protección, tareas de choque), b) funciones psicosociales (construcción de roles, aceptación y confirmación, consejería, amistad). La presencia de la función psicosocial presupone una relación de intimidad y vínculo interpersonal, mientras que la presencia de la función de carrera sugiere un instrumento restringido exclusivamente al crecimiento profesional (Kram \& Isabella, 1985). En este sentido, en el contexto universitario, las prácticas basadas simultáneamente en aspectos psicosociales y académicos contribuyen a la integración académica de los estudiantes y en los desafios que permean el proceso formativo. El modelo propuesto por Kram (1983) es frecuentemente utilizado como base conceptual en estudios acerca del proceso de mentoría en los más diferentes contextos.

Además de la no existencia de una fómula universal para las prácticas de tutoría y mentoría, otro aspecto marcado en la literatura hace referencia a las características de los tutores y los mentores. El profesor tutor aparece como actor esencial en el proceso formativo y en el desarrollo integral del alumno, para lo cual es importante su previa preparación metodológica, técnica y científica en el área de su disciplina, creatividad para la identificación y orientación en la solución de problemas de sus estudiantes, capacidad para la promoción del trabajo cooperativo, participación, interacción y responsabilidad crítica, y capacidad para la orientación en los distintos ámbitos de la formación del estudiante (Aguilar et al., 2012). Flores y colaboradores (2011) y Simão y colaboradores (2008) describen características personales propias de un buen tutor, entre las cuales destacan su habilidad comunicativa, dedicación, flexibilidad, paciencia, empatía, comprensión; fomentar la motivación en el grupo y el acercamiento constante a los alumnos. En cuanto al alumno mentor, normalmente este debe poseer 
excelentes resultados académicos, con habilidades interpersonales y interés por desarrollar exitosamente la responsabilidad del padrinazgo tutelar (Trimbur, 2008).

\section{Modalidades de la tutoría y la mentoría. Principales resultados descritos en la literatura}

Los programas de tutoría y mentoría se estructuran comúnmente, en dos modalidades: individual y grupal (Gómez-Collado; 2012; López et al., 2012). La modalidad grupal posee como aspecto positivo la inclusión de un número mayor de participantes, además de comprender costos menores y economía de tiempo. Por otro lado, en la modalidad individual, el estudiante recibe atención especial, según sus demandas específicas del alumno. A su vez, Borges y Carvalho (2013) afirman que esos programas pueden desarrollarse de manera formal e informal. Las tutorías y mentorías informales se caracterizan por las relaciones espontáneas, sin un tiempo definido de duración. Una tutoría o mentoría formal incluye acciones institucionales, normalmente contando con la estructuración de frecuencia, contenido y período de tiempo destinado a la intervención. La revisión de Borges y Carvalho (2013) sugiere que las relaciones informales poseen la ventaja de surgir a partir de una voluntad común entre los sujetos, lo que está directamente relacionado con la motivación de ambos; mientras que las relaciones formales son más favorables en cuanto a la accesibilidad, toda vez que la institución busca ofrecer el servicio a todos los estudiantes de nuevo ingreso. En esta dirección, algunos autores consideran que determinados programas planificados benefician a los alumnos generalmente menos propensos a tener un tutor o mentor informal, como las mujeres o las minorias étnicas (Kosoko-Lasaki, Sonnino \& Voytko, 2006; Santos \& Reigadas, 2002), o en otro caso, a recibir apoyo para lidiar con las exigencias académicas.

En cuanto a los resultados, el estudio internacional de GómezCollado (2012) sobre la percepción de los estudiantes referente a la 
experiencia de la mentoría, por ejemplo, indica que los sujetos involucrados en este proceso consideran el programa como una oportunidad para el intercambio y la elaboración del conocimiento, además de servir de enlace entre los indivíduos con otros miembros de la comunidad académica, y como forma de fortalecimiento de la solidaridad y el sentido de pertenencia, mediante las relaciones establecidas en la tutoría conjunta. El estudio de Wallace, Abel y Ropers-Huilman (2000) expone la comprensión de los significados que los estudiantes atribuyeron a sus experiencias como mentores. Los resultados de la investigación corroboran la hipótesis de que tales programas traen efectos positivos sobre las experiencias de los estudiantes. Entre los principales aspectos resaltados por los entrevistados se destacan el apoyo para vencer las dificultades académicas, y la percepción de no ser apenas un "número" dentro del nuevo contexto - o que favorece el sentido de pertenencia a la universidad.

En el estudio de de Santos y Reigadas (2002), en la cual los participantes fueron invitados a reflexionar sobre cómo se sentían antes y después de su inclusión en el proceso de mentoría, los principales resultados mostraron que con la participación en el programa los estudiantes experimentaron un aumento en la autoeficiencia, así como una mejor definición de metas académicas. En tanto, los graduandos acompañados por mentores de un mismo grupo étnico catalogan a estos como más eficientes en el desempeño profesional y personal, en comparación con los alumnos que eran asistidos por mentores de diferentes grupos étnicos; por último, la mayor frecuencia de contacto entre mentor y mentorado estuvo positivamente asociada com la adaptación del estudiante a la universidad, y con la satisfacción del mismo con el programa de mentoría. Kram y Isabella (1985) y Budge (2006) afirman que los mentores o tutores también pueden ser beneficiados en esa relación, tanto en los aspectos de satisfación y respeto, propios de la prestación de colaboración a otro individuo, como en el desarrollo de las habilidades interpersonales, además de otros aspectos como la paciencia, madurez, disponibilidad de tiempo y mayor responsabilidad. 
Otros estudios (Budge, 2006; Crisp \& Cruz, 2009) resaltan, por otro lado, que aunque exista una serie de investigaciones que señalan beneficios diversos proporcionados por tales programas, es notable la carencia de investigaciones científicas que potencien la crítica y la necesidad de lograr el perfeccionamiento y un mejor desempeño de estas prácticas, como por ejemplo, cuáles acciones específicas contribuyen significativamente en el desempeño académico, y qué grado de influencia tienen esos programas en lograr el éxito en el proceso formativo del estudiante. De forma igual manera, el estudio portugués de Simóes y Alarcão (2011), una revisión crítica de la literatura sobre la eficiencia de programas de mentoría, evidencio limitaciones metodológicas inherentes a las investigaciones, tanto en lo relativo a la pequeña dimensión de muchas de las muestras, como en la inexistencia del control experimental a la variabilidad del personal, en la mayor parte de las investigaciones, y la dependencia excesiva de medidas de autorrelato.

Según el artículo de Trimbur (2008), la relación jerarquizada entre mentor y mentorado puede ser incompatible con la relación horizontal entre pares, lo que en muchas veces puede generar malestar en una o ambas partes. El autor indica, como dificultad, una percepción de superioridad en relación con el mentorado, que puede ser superada en el proceso de formación y entrenamiento de mentores. El estudio de Simão y colaboradores (2008) indica que las principales dificultades percibidas por los nueve docentes entrevistados, responsables por la tutoría de proyectos interdisciplinarios, radican en la falta de experiencia del tutor, la dificultad de saber hasta dónde llega su intervención, cómo motivar un grupo para el tema específico del proyecto; asimismo, la acumulación de las funciones del tutor y docente, la falta de disponibilidad, las dificultades en la actualización permanente acerca de las diferenctes temáticas de búsqueda investigativa, y en la inexperiencia de los alumnos. Todos estos indicadores confirman la necesidad de una supervisión institucional en el desarrollo de esas intervenciones.

A partir de esta revisión, es claro el potencial beneficio de las estrategias institucionales de tutoría y mentoría para el desempeño integral del estudiante. En Brasil, en tanto, la discusión sobre estos tópicos 
aparece frecuentemente vinculada a la Educación a Distancia (Bernardini, 2012; Domeniquelli, 2010), muy poco sistematizada, por otro lado, en la Enseñanza Superior presencial. El hecho de que tales estrategias no existan de forma sistemática en las universidades brasileñas hasta el momento, "refuerza la idea de que cualquier iniciativa en este campo debe ser registrada, debatida y objeto de divulgación científica, favoreciendo la consolidación de buenas prácticas, la revisión de prácticas insatisfactorias y el progreso de ese campo específico de estudios sobre la universidad" (Sampaio, 2011, p. 226). Ante esta problemática, esta investigación privilegió la búsqueda en la literatura científica de experiencias en la tutoría y mentoría en universidades brasileñas, realizadas en carreras presenciales entre 1998 y 2013. Fue necesario identificar en cuáles áreas esos programas son más frecuentes, su configuración, objetivos, principales resultados y aspectos destacados por los autores, así como la pertinencia de los mismos, y de esa forma contribuir al conocimiento científico del tema. Además, con este trabajo pueden estimularse nuevas iniciativas de implementación de dichos programas en las IES brasileñas.

\section{Método}

Para esta revisión, se realizó una búsqueda bibliográfica en las bases de datos electrónicas SciELO, INDEXPsi y BVS-Psi, así como en el Banco de Tesis de CAPES, utilizando combinaciones dos a dos entre, por un lado, las palabras-clave tutoría, mentoría y padrinazgo; por outro, las palabras-clave universidad, enseñanza superior, estudiante universitario, graduación, alumno. Estas bases de datos fueron escogidas porque se presentan como las más completas para el acceso a la producción nacional en Psicología y áreas afines. 


\section{Resultados}

Las combinaciones de palabras generaron inicialmente un volumen total de 172 artículos, tesis de maestría y de doctorado, excluyendo las duplicaciones. Después de la lectura de los resúmenes, fueron también descartados los materiales no referidos a la enseñanza superior, estudios internacionales publicados en los periódicos nacionales, y los materiales relativos a procesos de enseñanza computacional. De esa selección (102), se excluyeron los estudios relativos a la educación superior a distancia. Finalmente, solo 31 hacen alusión a relatos de experiencia o investigación acerca de estos procesos en el ámbito de la graduación presencial universitária brasileńa, los que serán presentados con mayor detenimiento. La Tabla 1 caracteriza los estudios encontrados según rango de años de publicación, así como por autor, tipo de producción, tipo de estudio, objetivos y características de la muestra. Cabe señalar que no se encontraron publicaciones anteriores al ańo 2003.

Como se observa en la Tabla 1, la mayor parte de la producción vinculada a la tutoría y mentoría en la Enseñanza Superior brasileña se concentra entre 2011 y 2012 (38.70\%). De igual modo, se aprecia una significativa parcela de tesis de maestría (58.06\%), seguida por $32.25 \%$ de artículos y $9.67 \%$ de tesis de doctorado. Se percibe también que en el área de las Ciencias de la Salud (especialmente en la carrera de Medicina - 14 publicaciones) están las carreras para las cuales están más dirigidos los estudios sobre tutoría y mentoría. En cuanto a la región dedicada a este trabajo, los resultados se concentran principalmente en el sureste y noreste, con 15 y 10 publicaciones, respectivamente. Hubo también un predominio de trabajos en instituciones públicas (21 publicaciones). En la muestra analizada, 22 trabajos utilizaron el término 'tutoría' como acción desempeńada por un docente; dos estudios consideran la tutoría como relación desarrollada entre pares; y por último, siete trabajos utilizan el término "mentoria" para designar una acción efectuada por un docente orientador. Ninguno de los trabajos hizo referencia al concepto mentoría en tanto acción desarrollada entre pares. 


\section{Tabla 1}

Caracteristicas de los estudios realizados entre 2003 y 2012 por rango de años, autor, tipo de producción, tipo de estudio, objetivos y muestra

\begin{tabular}{cccccc}
\hline Año & $\begin{array}{c}\text { Autor } \\
(\mathrm{es})\end{array}$ & $\begin{array}{c}\text { Tipo de } \\
\text { producción }\end{array}$ & $\begin{array}{c}\text { Tipo } \\
\text { estudio }\end{array}$ & Objetivos & Muestra \\
\hline
\end{tabular}

De 2003 a 2009

2003 Bellodi Artículo Cuali- Investigar las representacio- Docentes de tativo nes asociadas al papel del Medicina tutor.

2004 Bellodi Artículo Cuali- Investigar las necesidades de Alumnos de et al. tativo los alumnos para el perfec- Medicina cionamiento del proceso de tutoría.

2005 Martin Tesis de Cuali- Describir las actividades de Estudiantes de maestría tativo los grupos PET y sus con- Química tribuciones a la Educación Superior.

2005 Medeiros Tesis de Cuali- Estudiar el impacto del Alumnos y maestría tativo programa de iniciación docentes de cientifica (PIBIC), en el Administración proceso formativo.

2005 Santos Tesis de Mixto Identificary analizar las Docentes de Júnior maestría funciones de mentoría. Administración

2007 Silva Tesis de Cuanti- Identificar funciones de Orientadores de maestría tativo Mentoría presentes en pos-graduación orientadores de Maestría y de Ciencias Doctorado Sociales

2008 Lourenço Artículo Cuali- Discurrirsobre la experen- Alumnos de tativo cia del PET-Psicología. Psicología

2008 Machado Tesis de Mixto Investigar el impacto de las Alumnos y maestría actividades basadas en el docentes de Método Tutoral de Apren- Farmacia dizaje

2008 Trenche Artículo Cuali- Analizar la implantación Alumnos y et al. tativo del nuevo proyecto Pedagógico, que incluye la tutoría Fonoaudiologia 


\begin{tabular}{|c|c|c|c|c|c|}
\hline Año & $\begin{array}{l}\text { Autor } \\
\text { (es) }\end{array}$ & $\begin{array}{c}\text { Tipo de } \\
\text { producción }\end{array}$ & $\begin{array}{l}\text { Tipo } \\
\text { estudio }\end{array}$ & Objetivos & Muestra \\
\hline 2009 & $\begin{array}{l}\text { Albuquer- } \\
\text { que et al. }\end{array}$ & Artículo & $\begin{array}{l}\text { Cuali- } \\
\text { tativo }\end{array}$ & $\begin{array}{l}\text { Investigar la adopción de } \\
\text { instrumentos viabilizadores } \\
\text { del proceso de enseñanza- } \\
\text { aprendizaje. }\end{array}$ & $\begin{array}{l}\text { Alumnos de } \\
\text { Odontologia }\end{array}$ \\
\hline 2009 & $\begin{array}{l}\text { Gui- } \\
\text { marães }\end{array}$ & $\begin{array}{l}\text { Tesis de } \\
\text { maestría }\end{array}$ & Mixto & $\begin{array}{l}\text { Investigar la existencia de } \\
\text { mentores y su influencia en } \\
\text { el proceso formativo. }\end{array}$ & $\begin{array}{l}\text { Alumnos de } \\
\text { Administración, } \\
\text { Marketing, y } \\
\text { Turismo }\end{array}$ \\
\hline 2009 & Oliveira & $\begin{array}{l}\text { Tesis de } \\
\text { maestría }\end{array}$ & $\begin{array}{l}\text { Cuali- } \\
\text { tativo }\end{array}$ & $\begin{array}{l}\text { Investigar las concepciones } \\
\text { de profesores y alumnos } \\
\text { sobre las sesiones tutorales. }\end{array}$ & $\begin{array}{l}\text { Docentes y } \\
\text { alumnos de } \\
\text { Medicina }\end{array}$ \\
\hline 2009 & $\begin{array}{l}\text { Perazzo } \\
\text { Filho }\end{array}$ & $\begin{array}{l}\text { Tesis de } \\
\text { maestría }\end{array}$ & $\begin{array}{l}\text { Cuali- } \\
\text { tativo }\end{array}$ & $\begin{array}{l}\text { Compreender el impacto de } \\
\text { la tutoría en el Aprendizaje } \\
\text { Basado en Problemas. }\end{array}$ & $\begin{array}{l}\text { Alumnos de } \\
\text { Medicina }\end{array}$ \\
\hline 2009 & Pinheiro & $\begin{array}{l}\text { Tesis de } \\
\text { maestría }\end{array}$ & $\begin{array}{l}\text { Cuali- } \\
\text { tativo }\end{array}$ & $\begin{array}{l}\text { Investigar la percepción de } \\
\text { los tutores sobre construcción } \\
\text { del conocimiento por los } \\
\text { estudiantes. }\end{array}$ & $\begin{array}{l}\text { Docentes de } \\
\text { Medicina }\end{array}$ \\
\hline 2009 & Prates & $\begin{array}{l}\text { Tesis de } \\
\text { maestría }\end{array}$ & $\begin{array}{l}\text { Cuali- } \\
\text { tativo }\end{array}$ & $\begin{array}{l}\text { Investigar la comprensión de } \\
\text { los alumnos sobre el proceso } \\
\text { tutoral en el Aprendizaje } \\
\text { Basado en Problemas. }\end{array}$ & $\begin{array}{l}\text { Alumnos de } \\
\text { Medicina }\end{array}$ \\
\hline 2009 & $\begin{array}{l}\text { Santana } \\
\text { et al. }\end{array}$ & Artículo & $\begin{array}{l}\text { Cuali- } \\
\text { tativo }\end{array}$ & $\begin{array}{l}\text { Reflexionar sobre las prácti- } \\
\text { cas de los docentes tutores }\end{array}$ & $\begin{array}{l}\text { Docentes } \\
\text { de Terapia } \\
\text { Ocupacional }\end{array}$ \\
\hline
\end{tabular}

De 2010 a 2012

\begin{tabular}{|c|c|c|c|c|c|}
\hline 2010 & Gomes & $\begin{array}{c}\text { Tesis de } \\
\text { doctorado }\end{array}$ & Mixto & $\begin{array}{l}\text { Analizar las habilidades } \\
\text { interpesonales a partir } \\
\text { de la tutoría. }\end{array}$ & $\begin{array}{l}\text { Etudiantes } \\
\text { de Medicina }\end{array}$ \\
\hline 2010 & Horta & $\begin{array}{l}\text { Tesis de } \\
\text { maestría }\end{array}$ & Mixto & $\begin{array}{l}\text { Analizar los diferentes } \\
\text { aspectos que influeyen } \\
\text { en la tutoria }\end{array}$ & $\begin{array}{l}\text { Tutores y } \\
\text { alumnos de } \\
\text { Medicina }\end{array}$ \\
\hline 2010 & $\begin{array}{l}\text { Leão } \\
\text { Pereira }\end{array}$ & $\begin{array}{l}\text { Tesis de } \\
\text { maestría }\end{array}$ & $\begin{array}{l}\text { Cuanti- } \\
\text { tativo }\end{array}$ & $\begin{array}{l}\text { Investigar la relación } \\
\text { entre bienestar, percep- } \\
\text { ción de necesidades y } \\
\text { búsqueda por los Pro- } \\
\text { gramas de Tutores }\end{array}$ & $\begin{array}{l}\text { Alumnos de } \\
\text { Medicina }\end{array}$ \\
\hline
\end{tabular}




\begin{tabular}{|c|c|c|c|c|c|}
\hline Año & $\begin{array}{l}\text { Autor } \\
\text { (es) }\end{array}$ & $\begin{array}{c}\text { Tipo de } \\
\text { producción }\end{array}$ & $\begin{array}{c}\text { Tipo } \\
\text { estudio }\end{array}$ & Objetivos & Muestra \\
\hline 2011 & $\begin{array}{c}\text { Francis- } \\
\text { chetti } \\
\text { et al. }\end{array}$ & Artículo & $\begin{array}{l}\text { Cuali- } \\
\text { tativo }\end{array}$ & $\begin{array}{l}\text { Analzar el role-playing } \\
\text { en tanto instrumento } \\
\text { pedagógico en la capaci- } \\
\text { tación tutoral docente. }\end{array}$ & $\begin{array}{l}\text { Docentes de } \\
\text { Medicina }\end{array}$ \\
\hline 2011 & Gonçalves & $\begin{array}{l}\text { Tesis de } \\
\text { maestría }\end{array}$ & $\begin{array}{l}\text { Cuali- } \\
\text { tativo }\end{array}$ & $\begin{array}{l}\text { Comprender las moti- } \\
\text { vaciones, recursos y } \\
\text { transformaciones relacio- } \\
\text { nadas con la tutoria. }\end{array}$ & $\begin{array}{l}\text { Docentes de } \\
\text { Medicina }\end{array}$ \\
\hline 2011 & Monte & $\begin{array}{l}\text { Tesis de } \\
\text { maestría }\end{array}$ & Mixto & $\begin{array}{l}\text { Investigar la percepción } \\
\text { de graduandos sobre las } \\
\text { funciones de mentoria } \\
\text { en la iniciación cien- } \\
\text { tifica. }\end{array}$ & $\begin{array}{l}\text { Alumnos del } \\
\text { IFRN }\end{array}$ \\
\hline 2011 & Tanji & $\begin{array}{c}\text { Tesis de } \\
\text { doctorado }\end{array}$ & $\begin{array}{l}\text { Cuali- } \\
\text { tativo }\end{array}$ & $\begin{array}{l}\text { Investigar las competen- } \\
\text { cias adquiridas por el } \\
\text { docente-tutor e impli- } \\
\text { caciones en la práctica } \\
\text { pedagógica. }\end{array}$ & $\begin{array}{l}\text { Docentes de } \\
\text { Enfermería }\end{array}$ \\
\hline 2011 & Urban & $\begin{array}{l}\text { Tesis de } \\
\text { maestría }\end{array}$ & $\begin{array}{l}\text { Cuali- } \\
\text { tativo }\end{array}$ & $\begin{array}{l}\text { Identificar y reflexionar } \\
\text { sobre el perfil del docente } \\
\text { a partir de la teoria de } \\
\text { mentoria de Kram. }\end{array}$ & $\begin{array}{l}\text { Alumnos y } \\
\text { egresados de } \\
\text { Carreras de la } \\
\text { FBV }\end{array}$ \\
\hline 2012 & Balau & $\begin{array}{l}\text { Tesis de } \\
\text { maestría }\end{array}$ & $\begin{array}{l}\text { Cuali- } \\
\text { tativo }\end{array}$ & $\begin{array}{l}\text { Identificar motivos de } \\
\text { inserción en el Programa } \\
\text { de Educación Tutoral } \\
\text { (PET), asi como las } \\
\text { transformaciones perci- } \\
\text { bidas por los mismos }\end{array}$ & $\begin{array}{l}\text { Alumnos } \\
\text { de Ciencias } \\
\text { biológicas, } \\
\text { Ciencias Exac- } \\
\text { tas, y Ciencias } \\
\text { Humanas }\end{array}$ \\
\hline 2012 & $\begin{array}{l}\text { Borba } \\
\text { Junior }\end{array}$ & $\begin{array}{l}\text { Tesis de } \\
\text { maestría }\end{array}$ & Mixto & $\begin{array}{l}\text { Investigar cómo las } \\
\text { metodologias activas } \\
\text { (entre las quales la } \\
\text { tutoria) influyen en el } \\
\text { proceso de enseñanza- } \\
\text { aprendizaje. }\end{array}$ & $\begin{array}{l}\text { Alumnos de } \\
\text { Medicina }\end{array}$ \\
\hline 2012 & Frison & Artículo & $\begin{array}{l}\text { Cuali- } \\
\text { tativo }\end{array}$ & $\begin{array}{l}\text { Evaluar la relación } \\
\text { entre actividades tuto- } \\
\text { rales y el proceso de } \\
\text { enseñanza-aprendizaje. }\end{array}$ & $\begin{array}{l}\text { Alumnos de } \\
\text { Pedagogía }\end{array}$ \\
\hline
\end{tabular}




\begin{tabular}{|c|c|c|c|c|c|}
\hline Año & $\begin{array}{l}\text { Autor } \\
\text { (es) }\end{array}$ & $\begin{array}{c}\text { Tipo de } \\
\text { producción }\end{array}$ & $\begin{array}{c}\text { Tipo } \\
\text { estudio }\end{array}$ & Objetivos & Muestra \\
\hline 2012 & $\begin{array}{l}\text { Gonçalves } \\
\text { \& Bellodi }\end{array}$ & Artículo & $\begin{array}{l}\text { Cuali- } \\
\text { tativo }\end{array}$ & $\begin{array}{l}\text { Comprender las } \\
\text { motivaciones y transfor- } \\
\text { maciones ocurridas con } \\
\text { la tutoría. }\end{array}$ & $\begin{array}{l}\text { Docentes de } \\
\text { Medicina }\end{array}$ \\
\hline 2012 & $\begin{array}{l}\text { Gonçalves } \\
\text { \& Bellodi }\end{array}$ & Artículo & $\begin{array}{l}\text { Cuali- } \\
\text { tativo }\end{array}$ & $\begin{array}{l}\text { Investigar Las dificul- } \\
\text { tades percibidas en la } \\
\text { tutoría, asi como los } \\
\text { recursos para enfren- } \\
\text { tarlas. }\end{array}$ & $\begin{array}{l}\text { Docentes de } \\
\text { Medicina }\end{array}$ \\
\hline 2012 & Ramalho & $\begin{array}{c}\text { Tesis de } \\
\text { doctorado }\end{array}$ & Mixto & $\begin{array}{l}\text { Evaluar el impacto del } \\
\text { programa de Tutoría } \\
\text { Especial de la UEPB en } \\
\text { el desempeño académico } \\
\text { y permanencia de los } \\
\text { discentes }\end{array}$ & $\begin{array}{l}\text { Alumnos de } \\
\text { pedagogía, } \\
\text { servicio social, } \\
\text { historia, } \\
\text { filosofía, comu- } \\
\text { nicación social, } \\
\text { derecho y física }\end{array}$ \\
\hline 2012 & Salgueiro & $\begin{array}{l}\text { Tesis de } \\
\text { maestría }\end{array}$ & $\begin{array}{l}\text { Cuali- } \\
\text { tativo }\end{array}$ & $\begin{array}{l}\text { Investigar la percepción } \\
\text { de orientandos y orien- } \\
\text { tadores sobre la relación } \\
\text { de orientación, a la luz } \\
\text { de la mentoria. }\end{array}$ & $\begin{array}{l}\text { Orientadores y } \\
\text { orientandos de } \\
\text { la UFPE }\end{array}$ \\
\hline
\end{tabular}

Algunas categorías fueron creadas para agrupar y permitir la mejor comprensión de los materiales encontrados: estudios relativos al método Aprendizaje Basado en Problemas (ABP) (ocho estudios); publicaciones con participantes de programas de iniciación científica (PIBIC) y programas de educación tutorial (PET) (cinco estudios); estudios centrados en la percepción de los alumnos (ocho), y la percepción de los profesores (seis estudios); estudios sobre programas de tutoría y mentoría en la postgraduación (dos) y la tutoría como herramienta para la producción de la escritura científica (dos). Los principales resultados serán descritos abajo, de acuerdo a esas categorías. 
a) Publicaciones referidas a programas de tutoria y mentoria relacionadas con la metodologia Aprendizaje Basado en Problemas (ABP)

Varios estudios discurren sobre actividades de tutoría relacionadas con la metodología denominada Aprendizaje Basado en Problemas $(\mathrm{ABP})$, muy utilizada en carreras en el área de Salud —especialmente en la Medicina- y que acostumbra contar con la participación de profesores tutores (Francischetti et al., 2011; Gomes, 2010; Horta, 2010; Oliveira, 2009; Perazzo Filho, 2009; Pinheiro, 2009; Prates, 2009; Tanji, 2011). Como aspectos positivos, los estudios observan que la propuesta formativa de la validación en la sesión tutoral es percibida como proceso, reflexiva, dialógica, diagnóstica, y hace énfasis en la posibilidad de feedback como factor motivador y determinante para solucionar las deficiencias detectadas y reforzar las potencialidades percibidas, además de aumentar la autonomía y el sentido crítico de los alumnos (Oliveira, 2009; Prates, 2009).

Tanji (2011) destaca el desarrollo de competencias en términos de conocimientos, habilidades y actitudes, así como la dimensión horizontal de la relación profesor-alumno dentro de la metodología ABP. Gomes (2010) investigó junto a estudiantes y docentes el desarrollo de las habilidades interpersonales en la formación médica, a partir de las sesiones tutoriales ABP, y los resultados sugieren la existencia de condiciones facilitadoras del proceso de comunicación como la aprehensión, empatía y sistematización de conocimientos. Horta (2010) observó que la recuperación de los conocimientos previos, la integración conceptual, el desarrollo de la autonomia en el proceso de aprendizaje, la interacción interpersonal y el proceso de validación, son facilitadores de la tutoría.

Son visibles las dificultades centradas en el desempeño de los docentes (falta de preparación), y los estudiantes (falta de madurez y sinceridad) (Oliveira, 2009). Pinheiro (2009) señala la resistencia de algunos docentes a la posibilidad de construir un conocimiento significativo durante el proceso tutorial, y sugiere que muchos profesores presentan preferencia por la praxis pedagógica tradicional. Francischetti y colaboradores (2011), en otra dirección, buscaron investigar 
junto a los docentes de la Facultad de Medicina de Marília el uso de la técnica role-playing (en la cual los profesores son incluidos en una situación-problema, en la que se realiza un cambio de roles (el docente asume el papel de discente) en tanto instrumento pedagógico en la tutoria relacionada com la ABP. Los docentes apuntarom fragilidades en las estratégias empleadas en la capacitación de los docentes para la conducción de un proceso de tutoría. Por otro lado, las innovaciones por medio de oficinas que se utilizaron de la técnica role-playing, proporcionaron un espacio reflexivo, de nuevas percepciones acerca de la importancia del programa de tutoría para el proceso de aprendizaje.

b) Publicaciones referentes a programas de tutoria y mentoria en los Programas de Iniciación Cientifica (PIBIC) y Programas de Educación Tutorial (PET)

El Programa de Educación Tutorial (PET) fue implantado en 1979 por CAPES. A partir de 2000, es coordinado por la Secretaría de Educación Superior (SESu/MEC). El PET se destina a apoyar alumnos de graduación, ofreciendo recursos que favorezcan su desarrollo académico autónomo (MEC, 2006) y procura integrar críticamente actividades de Enseñanza, Investigación y Extensión, bajo la dirección de un profesor tutor, en busca de alternativas para las estructuras curriculares convencionales. Tres estudios analizaron la función de la tutoría en estos programas.

Lourenço (2008), en un artículo descriptivo sobre la composición y las actividades de un grupo PET-Psicología, identificó que desde la implantación del programa, se observo un significativo envolvimiento de los alumnos becarios en actividades que representan preocupaciones con mejorías en la graduación. El autor señala que:

El modelo de educación tutorial posibilita la realización de actividades de carácter extracurricular que complementan la formación académica, haciendo que los alumnos asuman la responsabilidad sobre su propio desempeño y aprendizaje, además de permitir la ampliación de su visión del mundo y el dimensionamiento de su papel social (Lourenço, 2008, p. 100). 
Finalmente, identifica la importancia de ese modelo en un proyceto de calificación académica, especialmente en la formación del estudiante universitario. En la misma dirección, la tesis de maestría de Martin (2005) presenta un panorama general de lo que es un programa PET y analiza el proceso de formación de los becarios, utilizando como ejemplo las actividades de los integrantes del PET-Química de la UFPR. El análisis del proceso de formación mostró, según la autora, que la forma tutorial permite que el alumno elabore una relación de interioridad con sus actos y su producto permitiendo, de esta manera, un mejor conocimiento de su medio; eso contribuirá significativamente para su formación al estimular al alumno a reflexionar sobre sus propias actividades y sobre la relación entre esas actividades y el resultado de su formación. El estudio de Balau (2012) indentificó los motivos en torno a la decisión de ingreso del estudiante en un programa PET y los cambios percibidos por estos en su participación. Entre los principales resultados, las mayores razones para el ingreso se refieren a las categorías Oportunidad de desarrollo personal e interpersonal; ampliación de la formación académica y profesional; apoyo financiero; integración entre enseñanza, investigación y extensión; características positivas del grupo; e integración a la enseñanza superior. Al analizar los cambios apuntados por los estudiantes, fue posible percibir que los alumnos destacaron los puntos positivos en relación con los negativos, especialmente en lo que se refiere a la adquisición de habilidades, capacidades y competencias, conocimientos y composición de la red de contactos profesionales.

Los estudios que focalizan la relación tutorial en los programas de iniciación científica (Pibic) (Medeiros, 2005; Monte, 2011) buscaron entender las relaciones entre graduandos y orientadores a la luz de las teorías de la mentoría. Monte (2011) encontró resultados compatibles con los trabajos efectuados en otros países, en medios académicos. En la percepción de los contestadores ocurre un fornecimiento de las funciones de mentoría dentro del programa Pibic, en especial de las funciones modelo, tareas de choque, coaching y patrocinio. Entre las características encontradas para el profesor-orientador, indica el conocimiento y la experiencia, la disponibilidad, las relaciones interpersonales, la 
motivación y el liderazgo. Medeiros (2005) tuvo como objetivo estudiar el programa de iniciación científica del Centro Nacional de Desenvolvimiento Científico y Tecnológico en la carrera del graduando, a la luz de los fenómenos de mentoría y competencia. Los resultados revelaron que la iniciación científica contribuye positivamente a la carrera del graduando, afectando su desempeño en la graduación, en la maestria y en el mercado de trabajo. Fue notable, asimismo, que las relaciones de orientación son únicas, extremadamente personales y normalmente interactivas, además de presentar características típicas de mentoría.

\section{c) Publicaciones referentes a la validación de programas de tutoria y mentoria por los alumnos de diferentes carreras superiores}

En esta categoria se incluyen ocho estudios relativos a procesos de mentoría y tutoría que comparten como centro la percepción de los alumnos con relación a los desafios, demandas, e impacto de esos programas en su formación profesional. Los estudios de Guimarães (2009) y Urban (2011) se centraron en identificar la percepción de los alumnos en cuanto al perfil de profesores mentores, lo que contribuyen significativamente en su desarrollo humano y profesional. Los principales resultados de dos de las publicaciones indican la existencia de funciones de carrera y funciones psicosciales presentes en la relación de mentoría. Guimarães (2009), en este sentido, observa una ligera prevalencia de la primera categoria de funciones antes referida. Entre los resultados de Urban (2011) son mencionados aspectos como el dominio de contenido, didáctica, experiencia profesional, flexibilidad, empatía y buenas relaciones interpersonales en profesores que ejercen el papel de mentor. Por su parte, Frison (2012) investiga el impacto mutuo de la tutoría en un proceso de enseñanza-aprendizaje colaborativo de un semestre entre pares de alumnos de Pedagogía de una universidad pública. Los principales resultados sugieren que el proceso tutorial entre pares propició un aprendizaje colaborativo, activo, interactivo, mediado y autorregulado.

En otra direción, la tesis de doctorado de Ramalho (2012) se destaca por constituir una investigación acerca del impacto de un programa 
de tutoría especial de la Universidad Estadual de Paraíba (UEPB), destinado a alumnos con deficiencia. Las entrevistas indicaron la tutoría como herramienta positiva, que contribuye tanto a la permanencia de los estudiantes, como al exitoso desempeño académico. El estudio también apunta a la necesaria constante revisión, reformulación e incorporación de elementos que contemplen nuevas demandas en la dinámica del programa. De igual modo, el estudio de Albuquerque, Casotti, Souza y Souza (2009) indica la necesidad de un constante perfeccionamiento de esa herramienta pedagógica. Tales autores analizaron el impacto de la implantación del portafolio autorreflexivo y de la tutoría académica en la validación continua y formativa del proceso de enseñanza-aprendizaje de los alumnos de Odontología de una institución privada. La tutoría se configuraba como espacio de discusión sobre esos registros, proporcionando, así el permanente automonitoramiento del estudiante sobre su proceso de aprendizaje y desarrollo profesional y humano.

Bellodi, Massaroppe, Martins y Santos (2004), por su parte, investigaron las necesidades de los alumnos, a fin de subsidiar las intervenciones del Programa de Tutores en la Facultad de Medicina de la Universidad de São Paulo (FMUSP). Entre las principales demandas se destacan: 1) desempeño profesional; 2) Enseñanza-Aprendizaje; 3) relación médico-paciente; 4) ética médica; 5) ser alumno en medicina; 6) ser médico. Los resultados también confirman las diferentes necesidades de acuerdo con el período del curso en que el graduando se encuentra. Leấo Pereira (2010), investigo junto a alumnos del $6^{\circ}$ año de Medicina la relación entre bienestar, percepción de necesidades y búsqueda de asesoramiento. Los resultados indican que apenas alrededor de $1 / 3$ de los alumnos con ansiedad y depresión, o precario nivel de vida, buscaron los recursos institucionales de ayuda, entre los cuales la tutoría. En otro sentido, Borba Júnior (2012), en su estudio junto a los estudiantes de Medicina de la Universidade de Santa Cruz do Sul (UNISC), advirtió, en la percepción de los alumnos, dificultades al relacionar el aprendizaje significativo en las actividades de tutoría, con relación a las clases expositivas. 
d) Publicaciones referidas a la validación de programas de tutoría y mentoría por los profesores de diferentes carreras superiores

Aquí se incluyen seis publicaciones relativas a procesos de tutoría y mentoría, centradas en la percepción de los profesores en torno a los desafios, demanda y al impacto de esos programas en sus prácticas pedagógicas y en su desempeño profesional. Los estudios de Gonçalves (2011), Gonçalves y Bellodi (2012a) y de Gonçalves y Bellodi (2012b) hacen referencia a la investigación realizada con docentes tutores de la Facultad de Medicina de la Universidad de São Paulo (FMUSP), la cual indagó acerca de las motivaciones de los tutores, así como las transformaciones de estos indivíduos durante el desempeño de la tutoría. Asimismo, hizo énfasis en las dificultades y necesidades que estos presentaron, además de los principales recursos con que contaron para enfrentarlas. Los resultados indican dos categorías de motivaciones: aquellas relativas a los alumnos (el alumno actual o del tutor, en su condición de discente en el pasado) y a la institución (oficializar la función, colaborar con la formación, atualizarse sobre la facultad, retribuir oportunidades recibidas).

Entre las principales dificultades apuntadas por los docentes se señalan: duda en cuanto a las funciones y acciones que deben desempeñarse, insuficiente adhesión y poca participación de los alumnos en los encuentros de programa, además del tiempo limitado para el desarrollo exitoso de la relación entre tutor y estudiante. Los principales recursos utilizados para enfrentar dichas dificultades incluyen: recursos externos (coordinación y supervisión del programa e intercambio de experiencias y dificultades con otros tutores), y recursos internos (experiencia personal y profesional, así como el modo de enfrentar las dificultades). Reconocen que la tutoría no solo transforma a los alumnos, sino también a los propios tutores, quienes indican, entre los cambios, la oportunidad de perfeccionarse a sí mismos en su ámbito profesional, así como la experiencia del aprendizaje constante y las recompensas de su labor como docentes.

Bellodi (2003) investigó las representaciones del papel del tutor con profesionales de la FMUSP. Los resultados indicaron que existe 
predominio en la representatividad de tales prácticas, asimetría en la relación tutor-tutorado, relativa a los aspectos que incluyen los roles profesionales (como orientador y consejero), y los afectivo-familiares, tales como las figuras paternas y maternas. Las respuestas dirigidas a las relaciones simétricas, por su parte, se encaminaron a los papeles de vínculo, punto de referecia en la facultad, soporte, apoyo, protección, cuidado, y a otras características tales como compañerismo y amistad, disponibilidad y apertura. Santana, Kabbe, Carlo, Carretta y Elui (2009) son partidárias de la experiencia de la tutoría en tanto práctica que privilegia, por parte de los profesores, aspectos más allá del conocimiento técnico, en los cuales los alumnos deben ser reconocidos como entes activos en su proceso de formación. Santos Júnior (2005), que realizó un estudio exploratorio sobre la percepción de los profesores en torno al fenómeno de la mentoría, señala la importancia de su formalización entre profesores universitarios, válido para su perfeccionamiento profesional, lo cual trae como principales resultados el prevalecimiento de las funciones de carrera sobre el de las funciones psicosociales en la práctica de la mentoría.

\section{e) Publicaciones referidas a programas de tutoria y mentoría en la postgraduación}

La muestra total de textos analizados incluye dos investigaciones específicas vinculadas al trabajo de la mentoría en los cursos de postgrado. La base teórica de dichos estudios se sustenta desde la perspectiva de Kram (1985), ya mencionada en el acápite inicial de este trabajo. Ambos textos indican que la perspectiva de relación de orientación en la postgraduación puede ser vista como una relación de mentoría.

Salgueiro (2012) sugiere que a pesar de no existir definición regulada o consensuada en el programa, en torno a las prácticas de orientación en el postgrado, los interesados perciben que la relación de orientación supone un apoyo y un soporte que el orientador ofrece a su orientado, para alcanzar sus metas, ya sea durante un cronograma de objetivos a cumplir, o bien durante todo el proceso de tutelaje. No obstante, aunque la coordinación de responsabilidades propias de la orientación esté estrechamente vinculada con las funciones de carrera, 
se detectaron en las respuestas, tanto de orientadores como de orientados, funciones psicosociales en la relación. Aunque ambas funciones están presentes, las prácticas de la orientación inherentes a estas, en sentido general, no son sistemáticas y dependen mucho de las características individuales de los participantes. De igual modo, Silva (2007) buscó identificar funciones de mentoría y aspectos de liderazgo presentes en orientadores (de diversas instituciones) de Maestría y Doctorado en el área de Ciencias Sociales en Brasil. En los resultados se constató que la función de protección (carrera) y la función de amistad (psicosocial) no fueron contempladas, mientras que la función de tareas de choque (carrera) fue la más señalada. Silva (2007) concluye que existe falta de comprensión de lo que es el mentor por la mayoría de los encuestados (orientadores), quienes no identifican como elementos del modelo ideal de orientación varios de los comportamientos propios de las funciones de mentoría descritas por Kram (1985).

\section{f) La tutoría como herramienta para la producción y la investigación cientifica}

Otros dos estudios (Machado, 2008; Trenche, Barzaghi \& Pupo, 2008) articulan, como eje central de investigación, la tutoría en tanto herramienta para la producción del conocimiento científico. En el contexto del Método Tutorial de aprendizaje, Machado (2008) indagó en torno a la existencia de posibles dificultades sobre la producción de conocimiento científico, junto a alumnos y tutores de la carrera de Farmacia de una institución del DF. Mediante las observaciones a dos grupos tutoriales, aplicación de cuestionarios y entrevistas semi-estructuradas, el estudio indica que, aunque el proceso tutorial contribuye sin dudas a la formación de graduados, sus actividades no son todavía satisfactorias en lo relativo a la promoción y comprensión de la naturaleza de la producción científica por parte de los alumnos.

Por su parte, Trenche y colaboradores (2008) analizaron junto a docentes y estudiantes de la carrera de Fonoaudiología de la Pontifícia Universidad Católica de São Paulo (PUC-SP), el primer año de implantación del nuevo Proyecto Pedagógico, constituido por actividades tutoriales, seminarios, talleres técnicos, culturales y de escritura. 
Al enfatizar en la labor de tutoría, indica como eje central de trabajo la construcción del conocimiento científico para la formación profesional, a partir de los levantamientos bibliográficos de temáticas desarrolladas en el ámbito científico en el área de Fonoaudiologia. Los estudiantes validaron el trabajo de la tutoría, acompañada de otros dispositivos pedagógicos, catalogados como favorables para la inserción de estos a la carrera, su vínculo con los profesores y la profesión. Estos criterios posibilitaron ampliar sus puntos de vista con relación a estos tópicos.

\section{Discusión}

Este estudio tuvo como objetivo presentar una descripción de modelos y perspectivas con relación a la tutoría y mentoría universitarias, así como el de realizar un levantamiento bibliográfico sobre los programas existentes en la Enseñanza Superior brasileńa. A partir de los datos encontrados, es posible ofrecer algunas consideraciones.

Como punto de partida, es válido indicar que los procesos de mentoría y tutoría, en tanto herramientas pedagógicas encaminadas a beneficiar el proceso formativo del estudiante, se caracterizan por su diversidad y amplitud de acciones, según se observa tanto en la literatura científica foránea como en los estudios sobre esta temática en la Enseñanza Superior brasileña. Por ejemplo, las categorías de tutoría y mentoría referida a la metodologia Aprendizaje Basado en Problemas (ABP), Programa de Educación Tutoral (PET), programas de Iniciación Científica y tutoría como herramientas para la producción del conocimiento, se corresponden de modo más intrínseco al modelo académico de tutoría (Díaz, 2004; Rodríguez, 2004; Flores et al., 2011) - muy vinculado al legado universitario alemán- en el cual las actividades tutorales se centran en el dominio de los conocimientos académicos de sus estudiantes. Otras investigaciones nacionales se basan en modelos de tutoría y mentoría de carácter más heterogéneo, con énfasis no solo en el ámbito académico, sino también en las esferas vocacional y personal. Estas últimas se relacionan, de modo más íntimo con el modelo de 
Programas de tutoría y mentoría en universidades brasileñas / Albanaes et al.

desarrollo personal, propio de la tradición anglosajona (Bellodi, 2003; Guimarães, 2009; Santos Júnior, 2005; Santana et al., 2009; Urban, 2011).

En este sentido, al comparar los focos de atención de las actividades de mentoría y tutoría analizadas, de acuerdo con las definiciones entre funciones de carrera y funciones psicosociales descritas por Kram (1985), se puede observar en el país iniciativas que indican un ligero predominio de las funciones de carrera (Santos Júnior, 2005; Guimarães, 2009; Monte, 2011; Salgueiro, 2012), más centradas en las cuestiones y demandas específicas del aprendizaje, así como en la corrección de las dificultades académicas de los alumnos. Pudo observarse una insuficiente exploración, en las experiencias brasileñas, de las disímiles posibilidades que ofrece la tutoría, sobre todo en el ámbito psicosocial.

En la literatura consultada, la noción de mentoría aparece relacionada, prioritariamente, con las acciones desarrolladas entre pares (Erlich, 2010; Marinho-Araujo \& Bisinoto, 2011; Mundina et al., 2007). Por otra parte, en el análisis de los estudios en universidades brasileñas fueron encontrados apenas tres investigaciones (Santana et al., 2009; Frison, 2012; Ramalho, 2012) que consideran la posibilidad de desempeńo de tales prácticas con mentores o tutores que no poseen necesariamente un mayor status, grado jerárquico o autoridad. Tales publicaciones están direccionadas al modelo académico (Frison, 2012; Ramalho, 2012) y hacia el modelo formativo (Santana et al., 2009), descrito por Díaz (2004). En este estudio, especialmente el término 'mentoría' es conceptualizado como práctica desempeńada también por docentes, los cuales otorgan soporte de carrera y/o psicosocial, debido al vasto conocimiento y la experiencia que estos poseen (Kram, 1985). De este modo, converge con los resultados encontrados en las investigaciones de Monte (2011) y Urban (2011).

En Brasil, muchas carreras emplean el recurso del 'padrinazgo' como práctica de socialización de los alumnos de nuevo ingreso. Asimismo, la figura del mentor veterano es usual en la enseñanza superior, como lo demuestra el estudio sobre las actividades de acogida a ingresantes de una universidad pública brasileña, realizado por Bardagi, Luca 
y Girelli (2013). Ellos identificaron varias actividades de padrinazgo, caracterizadas como una relación informal de mentoría entre alumnos veteranos y alumnos noveles. Sin embargo, tales prácticas no son usualmente sistematizadas ni objeto de estudio, quedando asociadas a las relaciones informales entre colegas. De este modo, evaluar el impacto específico de dichas actividades entre pares podría ser una dirección para futuras indagaciones sobre la temática.

En relación con las modalidades de mentoría y tutoría, de acuerdo con los criterios consignados en la literatura internacional, se observó que la mayoría de los estudios nacionales analizados hacen referencia a prácticas esencialmente formales (Borges \& Carvalho, 2013) y grupales (Gómez-Collado; 2012; López et al., 2012). Al contrario, las publicaciones vinculadas a los programas de iniciación científica (PIBIC) y al postgrado, constituyen relaciones de mentoría esencialmente individuales. Por otro lado, existen poças informaciones en las publicaciones analizadas sobre aspectos como el tiempo de duración de estos procesos y las características específicas de los alumnos participantes, lo cual hace necesario una mayor sistematización de dichas informaciones. El predominio de los estudios cualitativos también fue notable en las publicaciones analizadas. La tentativa de ampliación de las muestras y la consideración de medidas más objetivas y de mayor alcance, en nuevos estudios, pueden contribuir a una mejor comprensión del impacto que alcanzan los programas de tutoría y mentoría en el sistema formativo brasileño. Incrementar el control sobre las variables que intervienen en el proceso, identificar con claridad las características de los participantes, así como las etapas en el proceso, son pasos a seguir para conocer, como indican Simóes y Alarcão (2011), a eficacia de los programas de mentoría y tutoría.

Como impactos propios de la participación en programas de tutoría y mentoría, los estudios analizados también convergen con la literatura (Gómez-Collado, 2012; Santos \& Reigadas, 2002; Wallace et al., 2000), indicando un predominio de resultados positivos de esas práticas, según las experiencias descritas, tales como: la acción de apoyo para lograr la permanencia, el éxito y la integración académicas; la opor- 
tunidad de desempeñarse personal y profesionalmente; el desarrollo de la autonomía en el proceso de aprendizaje y la horizontalización de las relaciones profesor-alumno, lo cual propicia una retroalimentación de dicha práctica, de forma colaboral, activa, interactiva, mediada y autorregulada. Las investigaciones analizadas indican, además, la posibilidad de que no solo los alumnos tutorados o mentorados obtengan benefícios, sino también los docentes tutores o mentores (Prates, 2009; Gonçalves, 2011; Gonçalves \& Bellodi, 2012a, entre otros), de acuerdo con lo señalado por la literatura científica internacional.

La investigación en torno a las cuestiones críticas en la conformación de programas de tutoría y mentoría deben ser de igual forma señaladas, toda vez que tales registros influyen en el perfeccionamiento de esas herramientas pedagógicas. En este sentido, el análisis de las publicaciones nacionales indica que los tutores y mentores en las universidades brasileñas presentan dificultades en su formación, entre las que se encuentran: dudas en cuanto a las funciones y acciones que pueden desempeñar y el limitado tiempo que poseen para desarrollar la relación entre tutor y estudiante (Gonçalves, 2011; Gonçalves \& Bellodi, 2012b; Gonçalves \& Bellodi, 2012a), así como la incomprensión en torno a sus roles como mentor (Silva, 2007).

Los estudios centrados en las valoraciones sobre las características inherentes a un tutor o mentor ideal (Monte, 2011; Urban, 2011) corroboran lo señalado por la literatura respecto a la necesidad de la preparación metodológica (Aguilar et al., 2012), y de la flexibilidad y la empatía (Simão et al., 2008; Flores et al., 2011). Entre tanto, algunos tutores entrevistados manifestaron su desinterés sobre tales procesos tranformadores de la enseñanza (Gonçalves, 2011; Gonçalves \& Bellodi, 2012b; Gonçalves \& Bellodi, 2012a), pues son partidarios de la praxis pedagógica tradicional (Pinheiro, 2009). Esto es reflejo, por un lado, de la poca disponibilidad de docentes para la práctica de la mentoría; por otro, de la falta de preparación y sensibilización de los profesores para actuar como mentores/tutores.

En relación con este último aspecto fueron señaladas, además, otras causas: la insuficiente adhesión de determinados grupos de alumnos 
a la modalidad de tutoría (Leão Pereira, 2010), la poca participación del estudiantado en los encuentros del programa (Gonçalves, 2011), así como la dificultad de vincular el aprendizaje en las actividades de tutoría académica con las restantes materias (Borba Júnior, 2012). Esos aspectos confirman el papel crucial de una formación previa adecuada de los tutores y mentores para la conformación de relaciones de tutoría y mentoria, que promuevam el efectivo desempeño profesional y personal del estudiante en su proceso formativo. Francischetti y colaboradores (2011), por ejemplo, indican la existencia de frágiles estrategias utilizadas en la capacitación de los docentes para la conducción de un proceso de tutoría, y que la implementación de nuevas estrategias de preparación a esos indivíduos posibilitó un cambio en sus percepciones acerca de la importancia del programa de tutoría para el proceso de aprendizaje. Considerando, por tanto, el protagonismo de los mentores y tutores en estos programas, es fundamental la reflexión en torno a la necesidad de formar los sujetos encargados de conducir los programas junto a los alumnos, a través de la mediación del desarrollo de competencias sociales y relacionales, conforme apuntan Marinho-Araujo y Bisinoto (2011) y Budge (2006).

Primero que todo, es esencial que mentores y tutores sean eficazmente orientados, a fin de esclarecer cuestiones relativas al desempeño administrativo, la toma de conciencia de las funciones que desarrollarán, las estrategias que contribuyan a una exitosa relación entre mentores y tutores, además de disponer del tiempo necesario para enfrentar y solucionar las dudas y dificultades relativas a estos programas. Es importante también atender a la noción de que el proceso de interación con los estudiantes y sus demandas constituyen elementos en constante transformación. Por ello es necesario su revisión, reformulación y el permanente perfeccionamiento de esta herramienta pedagógica (Albuquerque et al., 2009; Ramalho, 2012) por parte de los indivíduos responsables por el desempeño de tales prácticas.

En sentido general, este estudio de levantamiento revela como elemento positivo la existencia de experiencias de tutoría y mentoría en carreras diversas en la Enseñanza Superior brasileña, aunque es notable 
un predominio de esos programas, sobre todo, en el área de las Ciencias de la Salud —especialmente en la carrera de Medicina, en el cual la tutoría vinculada a la metodologia Aprendizaje Basado en Problemas $(\mathrm{ABP})$ es una práctica tradicional. Considerando los posibles benefícios para discentes e instituciones —en relación con el éxito e integración académica - todavía prevalecen pocos ejercicios de sistematización de dichas prácticas en las universidades. Ante el desconocimiento de esas herramientas pedagógicas por muchos profesores e investigadores en la Enseñanza Superior brasileña, la divulgación de esta importante temática, mediante artículos científicos, resulta útil, teniendo en cuenta el fácil acceso y la disponibilidad con que se cuenta a través de tesis de maestría y de doctorado — predominantes en la muestra analizada.

Considerando el actual escenario en el que inserta la Enseńanza Superior brasileña, en la que existe una mayor diversidad de alumnos y, por consiguiente, mayores y variadas necesidades en ellos, en términos de adaptación y aprendizaje exitoso, es necesario reflexionar en torno a la implementación de estrategias eficaces, entre las cuales la tutoría y mentoría pueden ser ejemplos interesantes, pues ayudan al estudiante en su proceso de integración a la universidad. En este sentido, es importante también concederle mayor atención a los resultados alcanzados por esos programas en alumnos deficientes y en aquellos que pertenecen a minorías (Kosoko-Lasaki et al., 2006; Santos \& Reigadas, 2002), así como hacer énfasis en la necesidad de potenciar aspectos como los psicosociales, vocacionales y académicos, en un contexto acompańado de nuevas exigencias a los estudiantes.

\section{Referencias}

Aguilar, N. A., Rodríguez, C. M. \& Bugdug, A. T. (2012). La interacción tutor - estudiante en la Educación Superior. Un acercamiento a su diagnóstico. Humanidades Médicas, 12(3), 409-426.

Albuquerque, C. J. M., Casotti, E., Souza, M. C. A. \& Souza, T. C. (2009). Instrumentos inovadores da prática pedagógica: uma 
experiência no ensino de odontologia. Revista fluminense de Odontologia, 15(32), 49-53.

Balau, M. M. N. (2012). A experiência no programa de educação tutorial (PET) e a formação do estudante do ensino superior (Tesis de Maestría no publicada). Universidade Estadual de Campinas, Campinas.

Bardagi, M. P., Luca, G. G. \& Girelli, S. (2013). Caracterização das atividades de acolbimento ao aluno ingressante da UFSC: A lacuna da avaliação. Anais do VI Congresso Brasileiro de Avaliação Psicológica, Maceio.

Bellodi, P. L. (2003). O que é um tutor? Representaçóes do papel em um grupo de professores de medicina durante o processo de seleção. Revista Brasileira de Educação Médica, 27(3), 205-212.

Bellodi P. L., Martinho T., Massaroppe, T. \& Martins M. A. (2004). Temas para um Programa de Tutoria em Medicina: uma investigação das necessidades dos alunos da FMUSP. Revista Brasileira de Educação Médica, 28(2), 89-184.

Bernardini, I. S. (2012). Gestão na educação a distância: o contexto dos processos focados nas pessoas (Tesis de Maestría no publicada). Universidade Federal de Santa Catarina, Florianópolis.

Borba Júnior, A. M. (2012). Aprendizagem Significativa em Fisiologia Humana. Ausubel e a Formação de Conceitos Básicos (Tesis de Maestría no publicada). Universidade de Santa Cruz do Sul, Santa Cruz do Sul.

Borges, L. O. \& Carvalho, V. D. (2013). Tutorização organizacional de novos empregados. En L. O. Borges \& L. Mourão (Orgs.), $O$ trabalho e as organizaçôes: atuaçóes a partir da Psicologia. (1 ed., pp. 406-432). Porto Alegre: Artmed.

Budge, S. (2006). Peer Mentoring in Post- Secondary Education: Implications for Research and practice. Journal of College Reading and Learning, 37(1), 73-87.

Crisp, G. \& Cruz, I. (2009). Mentoring college students: a critical review of the literature between 1990 and 2007. Research in Higher Education, 50, 525-545. 
Díaz H., D. P. (2004). La tutoría académica en la educación universitaria: una experiencia en el núcleo de Actividad Física, del área de Movimiento y Postura, en el programa de Medicina de la Universidad de Antioquia. Iatreia, 17(4), 404-413.

Domeniquelli, A. M. T. (2010). A relação pedagógica na tutoria a distância: um estudo a partir do curso de pedagogía (Tesis de Maestría no publicada). Universidade Metodista de São Paulo, São Bernardo do Campo.

Eby, L. T., Allen, T. D., Evans, S. C., Ng, T. \& DuBois, D. L. (2008). Does mentoring matter? A multidisciplinary meta-analysis comparing mentored and non-mentored individuals. Journal of Vocational Behavior, 72, 254-267.

Erlich, P. (2010). Mentoria e adesão à atividade física: o caso dos usuários da academia Chesf(Tesis de Maestría no publicada). Centro de Pesquisa e Pós-Graduação em Administração, Faculdade Boa Viagem, Recife.

Flores, G. C., Kury, E. C. \& Abreu, L. F. (2011). Tutoría en educación superior: una revisión analítica de la literatura. Revista de la educación superior, 1(157), 189-209.

Francischetti, I., Corrêa, A. C. L., Vieira, C. M., Lazarini, C. A., Rolin, L. M. G. \& Soares, M. O. M. (2011). Role-playing: estratégia inovadora na capacitação docente para o processo tutorial. Interface - Comunicação, Saúde, Educação, 15(39), 1207-1218.

Frison, L. M. B. (2012). Tutoria entre estudantes: uma proposta de trabalho que prioriza a aprendizagem. Revista Portuguesa de Educação, 25(2), 217-240.

Geib, L. T. C., Krahl, M., Poletto, D. S., \& Silva, C. B. (2007). A tutoria acadêmica no contexto histórico da educação. Revista Brasileira de Enfermagem, 60(2), 217-220.

Gomes, M. M. (2010). Habilidades Interpessoais no Processo Educativo em Medicina: a Comunicação em Sessóes Tutoriais (Tesis de Maestría no publicada). Universidade Federal de São Paulo, São Paulo. 
Gómez-Collado, M. E. (2012). La percepción de los estudiantes sobre el Programa de Tutoría Académica. Convergencia: Revista de Ciencias Sociales, 58, 209-233.

Gonçalves, M. C. N. (2011). Vivências de tutor: estudo qualitativo na abordagem da psicologia analitica (Tesis de Maestría no publicada). Universidade de São Paulo, São Paulo.

Gonçalves, M. C. N. \& Bellodi, P. L. (2012a). Ser mentor em medicina: uma visão arquetípica das motivações e transformaçōes na jornada. Interface - Comunicação, Saúde, Educação, 16(41), 501-514.

Gonçalves, M. C. N. \& Bellodi, P. L. (2012b). Mentors also need support: a study on their difficulties and resources in medical schools. São Paulo Medical Journal, 130(4), 252-258.

Guimarães, F. D. (2009). Mentoria: Professores-mentores e a percepção desses a respeito do papel da mentoria em sua formaçáo e atuação: o caso da FACIPE - uma Instituição de Ensino Superior Privada de Pernambuco (Tesis de Maestría no publicada). Centro de Pesquisa e Pós-Graduação em Administração, Faculdade Boa Viagem, Recife.

Horta, F. M. B. (2010). A Sessão Tutorial na Abp do Curso Médico da Unimontes: Desafios e Possibilidades (Tesis de Maestría no publicada). Universidade Federal de São Paulo, São Paulo.

Kosoko-Lasaki, O., Sonnino, R. E. \& Voytko, M. L. (2006). Mentoring for women and underrepresented minority faculty and students: experience at two institutions of Higher Education. Journal of the national medical association, 98(9), 1449-1459.

Kram, K. E. (1983). Phases of the mentor relationship. Academy of Management Journal, 26(4), 608-625.

Kram, K. E. \& Isabela, L. A. (1985). Mentoring alternatives: The role of peer relationships in career development. Academy of Management Journal, 28(1), 110-132.

Leão Pereira, P. B. O. S. (2010). Bem-estar e busca de ajuda: um estudo junto a alunos de Medicina ao final do curso (Tesis de Maestría no publicada). Universidade de São Paulo, São Paulo. 
López, R. I. G., Salazar, O. C., García, J. J. V. \& Medina, I. R. C. (2012). Impacto del Programa de Tutoría en el desempeño académico de los alumnos del Instituto Tecnológico de Sonora. Revista Electrónica de Investigación Educativa, 14(1), 106-121.

Lourenço, L. M. (2008). O PET-Psicologia na Universidade Federal de Juiz de Fora. Psicologia em Pesquisa, 2(1), 98-100.

Machado, N. H. S. (2008). O ensinar e o aprender a fazer pesquisa: o real e o desejado. (Tesis de Maestría no publicada). Universidade de Brasília, Brasília.

Marinho-Araujo, C. M. \& Bisinoto, C. (2011). Psicologia escolar na educação superior: construindo possibilidades diferenciadas de atuação. En R. S. L. Guzzo \& C. M. Marinho-Araujo (Orgs.), Psicologia Escolar: identificando e superando barreiras (1ed., pp. 193-214). Campinas: alínea.

Martin, M. G. M. B. (2005). O Programa de Educação tutorial-PET: Formação ampla na graduação [Tesis de Maestría no publicada]. Programa de Pós-Graduação em Educação, Universidade Federal do Paraná. Curitiba.

Medeiros, R. A. S. M. (2005). O Impacto do programa de iniciação cientifica (CNPq) na carreira do graduando, à luz dos fenômenos de mentoria e de competência: o caso dos alunos do curso de Administração da UFPE (Tesis de Maestría no publicada). Universidade Federal de Pernambuco, Recife.

Ministério da Educação y Secretaria de Educação Superior (2006). Programa de Educação Tutorial PET. Manual de orientaçóes básicas. Brasília, DF: Secretaria de Educação Superior.

Monte, G. T. (2011). Prática da Mentoria: Estudo de caso no Instituto Federal de Educação, Ciência e Tecnologia do RN (Tesis de Maestría no publicada). Universidade Potiguar, Natal.

Mundina, J. B., Pombo, N. C. \& Ruiz, E. R. (2007). Dimensión convergente de la tutoría en la universidad: tutoría entre iguales. Recuperado de http://www.eduonline.ua.es/jornadas2007/ comunicaciones/2G3.pdf 
Oliveira, V. T. D. (2009). Avaliação na Sessão Tutorial do Curso de Medicina da Unimontes: Apreendendo Concepçóes e Práticas (Tesis de Maestría no publicada). Universidade Federal de São Paulo, São Paulo.

Perazzo Filho, B. (2009). O Conhecimento tácito e aprendizagem baseada em problemas no curso de medicina da UESB: aproximaçóes e reflexóes (Tesis de Maestría no publicada). Universidade Federal da Bahia, Salvador.

Pinheiro, C. C. O. (2009). A atuação do professor na construção do conhecimento dos estudantes durante o processo tutorial no Curso de Medicina da UESB: a visão do professor-tutor. (Tesis de Maestría no publicada). Universidade Federal da Bahia, Salvador.

Prates, M. E. V. O. (2009). O processo tutorial no método de aprendizagem baseada em problemas (ABP) no curso de Medicina da UESB: a compreensão dos estudantes. (Tesis de Maestría no publicada). Universidade Federal da Bahia, Salvador.

Ramalho, M. N. (2012). A inclusäo de alunos com deficiência na UEPB: uma avaliação do programa de tutoria especial (Tesis doctoral no publicada). Universidade do Estado do rio de Janeiro, Rio de Janeiro.

Rodríguez, S. (2004). Manual de tutoría universitária: Recursos para la accion. Barcelona: Octaedro.

Rubio, P. P. \& Martinez, J. F. (2012). La acción tutorial desde la perspectiva de los alumnos de la Universidad Autónoma de San Luis Potosí. Perfiles educativos, 34(138), 28-45.

Salgueiro, J. C. F. (2012). A Prática da Orientação, em Programa de Pós-Graduaçáo Stricto Sensu, à Luz dos Fundamentos e Princípios da Mentoria: a experiência de Orientadores e Orientandos do Programa de Pós-Graduação em Administração da UFPE (Tesis de Maestría no publicada). Universidade Federal de Pernambuco. Recife.

Sampaio, S. (2011). A educaçáo superior como espaço privilegiado para a orientação acadêmica. En R. S. L. Guzzo \& C. M. Marinho- 
Araujo (Orgs.), Psicologia Escolar: identificando e superando barreiras (1ed., pp. 215-228). Campinas: Alínea.

Santana, C. S., Kebbe, L. M., Carlo, M. M. R. P., Carretta, R. Y. D. \& Elui, V. M. C. (2009). Reflexóes sobre a prática de tutoria com estudantes de terapia ocupacional. Trabalho, Educação e Saúde, 7(1), 167-182.

Santos Júnior, L. S. (2005). O fenômeno da mentoria na percepção dos professores do curso de administração da Faculdade Integrada do Recife: insights para um modelo brasileiro de mentoria (Tesis de Maestría no publicada). Universidade Federal de Pernambuco, Recife.

Santos, S. J. \& Reigadas, E. T. (2002). Latinos in Higher Education: an evaluation of a university faculty mentoring program. Journal of Hispanic Higher Education, 1(1), 40-50.

Silva, L. B. (2007). Orientação de Mestrado e de Doutorado, à Luz dos Construtos de Mentoria e Liderança (Tesis de Maestría no publicada). Faculdade Boa Viagem, Recife.

Simão, A. M. V., Flores, A., Fernandes, S. \& Figueira, C. (2008). Tutoria no ensino superior: concepçóes e práticas. Sisifo - Revista de ciências da educação, 7, 75-88.

Simões, F. \& Alarcão, M. (2011). A eficácia da mentoria escolar na promoção do desenvolvimento socioemocional e instrumental de jovens. Educação e Pesquisa, 37(2), 339-354.

Tanji, S. (2011). As competências do docente tutor no contexto da mudança curricular do curso de graduação em enfermagem no UNIFESO (Tesis doctoral no publicada). Universidade Federal do Rio de Janeiro, Rio de Janeiro.

Trenche, M. C. B., Barzaghi, L. \& Pupo, A. C. (2008). Mudança curricular: construção de um novo projeto pedagógico de formação na área da Fonoaudiologia. Interface - Comunicação, Saúde, Educação, 12(27), 697-711.

Trimbur, J. (2008). Peer Tutoring: A Contradiction in Terms? En R. W. Barnett \& J. S. Blumner (Orgs.), The Longman Guide to Wri- 
ting Center Theory and Practice. (288-295). Nueva York: Pearson Education.

Urban, R. C. (2011). Mentoria, Professores e Educação: Um estudo de caso na Faculdade Boa Viagem (Tesis de Maestría no publicada). Centro de Pesquisa e Pós-Graduação em Administração, Faculdade Boa Viagem. Recife.

Wallace, D., Abel, R. \& Ropers-Huilman, B. (2000). Clearing a path for success: deconstructing borders through undergraduate mentoring. The Review of Higher Education, 24(1), 87-102.

Recibido: 17 de mayo, 2014 Aceptado: 05 de agosto, 2014 